\title{
El diagnóstico psicopedagógico y el comportamiento infantil. Estudio de caso
}

\author{
Psychopedagogical diagnosis and child behavior: case study \\ Diagnóstico psicopedagógico e comportamento infantil: estúdio de caso
}

\section{RESUMEN}

En la actualidad existen problemas de comportamiento en los niños dentro de las aulas escolares, que como experiencia proviene de la formación que reciben de sus padres como educadores. Este estudio parte sobre diagnóstico psicopedagógico y el comportamiento infantil en una Unidad Educativa durante el periodo 2018-2019", el cual establecelaimportancia del diagnóstico psicopedagógico en el comportamiento infantil. La metodología fue descriptiva y analítica, se aplicaron registros anecdóticos y escala descriptiva, misma que permitió conocer la problemática planteada. Se presentó una propuesta para mejorar el comportamiento infantil y el área socio afectivo. Los niños con problemas de conducta suelen mostrarse desobedientes, malcriados, berrinchudos y groseros, el diagnóstico psicopedagógico permite conocer las dificultades que se presenten en el comportamiento de los pequeños desde edades tempranas, buscar una solución para evitarlas y utilizar estrategias metodológicas acordes a sus diferencias individuales, al entorno social, realidad socioeconómica y la creatividad del docente.

Palabras clave: $\begin{aligned} & \text { Diagnóstico } \\ & \text { psicopedagógico; }\end{aligned}$
infantil; Desarrollo; Socio afectividad

Currently there are behavioral problems in children in school classrooms, which as an experience comes from the training they receive from their parents as educators. This study starts on psycho-pedagogical diagnosis and child behavior in an Educational Unit during the period 20182019", which establishes the importance of psycho-pedagogical diagnosis in child behavior. The methodology was descriptive and analytical, anecdotal records and descriptive scale were applied, same that allowed to know the raised problematic. A proposal was presented to improve children's behavior and the socio-affective area. Children with behavioral problems tend to be disobedient, spoiled, tantrums and rude. The psycho-pedagogical diagnosis allows to know the difficulties that arise in the behavior of children from an early age, to find a solution to avoid them and to use methodological strategies according to their individual differences, the social environment, socioeconomic reality and the creativity of the teacher.

Key words: Psychopedagogical diagnosis; Child behavior; Development; Socioaffectivity

\section{RESUMO}

Atualmente existem problemas comportamentais em crianças nas salas de aula da escola, que como experiência vem do treinamento que elas recebem de seus pais como educadores. Este estudo começa com o diagnóstico psicopedagógico e o comportamento das crianças em uma Unidade Educacional durante o período 2018-2019”, que estabelece a importância do diagnóstico psicopedagógico no comportamento das crianças. A metodologia foi descritiva e analítica, foram aplicados registros anedóticos $\mathrm{e}$ uma escala descritiva, o que nos permitiu compreender os problemas levantados. Foi apresentada uma proposta para melhorar o comportamento das crianças e a área sócio-afetiva. As crianças com problemas de comportamento tendem a ser desobedientes, mimadas, birras e rudes. $\mathrm{O}$ diagnóstico psicopedagógico nos permite conhecer as dificuldades que surgem no comportamento das crianças desde cedo, procurar uma solução para evitálas e utilizar estratégias metodológicas de acordo com suas diferenças individuais, o ambiente social, a realidade sócioeconômica e a criatividade do professor.

Palavras-chave:

Diagnóstico psicopedagógico; Comportamento infantil; Desenvolvimento; Sócio-afetividade 


\section{INTRODUCCIÓN}

En la actualidad la relación entre padres e hijos es fundamental en el desarrollo de hábitos, conductas y comportamientos de todo ser humano, por ello desde muy pequeños los primeros educadores en este caso los padres deben cuidar su comportamiento, dado que el reflejo de ellos será lo que los infantes imiten. La educación formal de los niños interviene en este proceso de formación enseñando y reforzando una serie de habilidades y destrezas necesarias para su desenvolvimiento en la sociedad; además son esenciales para lograr personas integras, por tanto, la estimulación de capacidades cognitivas, psicomotrices, emocionales y sociales del adulto son el resultado del proceso formativo desde la niñez.

La persona encargada de guiar todo este proceso es el educador/a, él/ella puede evidenciar la evolución del educando dentro del proceso educativo, observar cuales son las asignaturas en el que se puede desenvolver mejor y también conocer las falencias que el estudiante tiene. Por este motivo la pedagogía conjuntamente con la psicología desarrolla una herramienta de diagnóstico esencial para el docente, ya que a través de ella se puede conocer, detectar y tratar problemas en todos los alumnos, en cualquier etapa de la vida, sin embargo, se trabaja más en la edad escolar, puesto que es importante el rendimiento académico, mejorando y potencializando la calidad y capacidad de aprendizaje de los niños.

El diagnóstico es el punto de llegada de un proceso complejo, $y$, al mismo tiempo, el punto de arranque para mejorar educación o reducción del caso analizado. Podemos, pues, definir el diagnostico en psicología como un proceso general de identificación y valoración de las características de comportamiento, aptitudes y actitudes $\mathrm{o}$ atributos personales de un individuo en un contexto dado. (Mora, 1998).

Por ende, el comportamiento infantil es una necesidad del niño o como consecuencia de la etapa del desarrollo en el que se forma; su conducta, temperamento y la interacción con las demás personas. La mayor parte de los comportamientos infantiles son aprendidos, dado que, al nacer el niño desconoce de normas y pautas de conducta que se consideren adecuadas, por tal motivo él busca modelos a seguir, aprende y pone en práctica lo que ve de ellos.

Es porque ello, que este estudio plantea determinar la importancia del diagnóstico psicopedagógico en el comportamiento infantil en el subnivel de inicial 2 (4 a 5 años) de la Unidad Educativa "El Carmen", ya que para la mayoría de los padres de familia y profesionales de la primera infancia si principal misión es lograr que el pequeño se comporte de una forma socialmente aceptable, adecuada, justa y solidaria. De igual manera, la investigación tiene como propósito ampliar la argumentación sobre el nivel de desarrollo biopsicosocial de la niña, al mismo tiempo establecer la relación que existe entre el diagnóstico psicopedgógico y el comportamiento infantil; $y$, proponer una guía de estrategias didácticas para mejorar el comportamiento infantil en los niños de educación inicial de 4 a 5 años.

Para ello, es necesario, guiar el comportamiento de los niños mediante un proceso que les permita construir una conducta positiva. Esta mejora enfoca su atención no solo en la disciplina a través de la obediencia y control, sino que también debe incluir 
una guía para el comportamiento, un conjunto de procesos en donde los niños aprendan a dominar y regir ese comportamiento para llegar a ser independientes y autosuficientes. (Morrison, 2005). El comportamiento de los niños dentro del aula de clases puede ser distinto al del hogar, quizás esto se deba que aun ellos no se sientan en plena confianza con el docente para expresarse como son, pero es el educador quien conoce, observa y analiza el desarrollo biopsicosocial y comportamental de sus estudiantes, puede conocer cuáles son las virtudes, defectos y problemas.

Lo fundamental a la hora de orientar al niño es saber cómo son, es decir, como crecen y se desarrollan. Por adversidad no todos los maestros tienen la capacidad de guiar al estudiante, quizás por falta de preparación, interés, orientación o capacitación. En consideración al enunciado se justifica la importancia del presente artículo, ya que el mismo luego de una minuciosa búsqueda de teoría y análisis de los resultados, se ofrece una propuesta que pretende disminuir la problemática, tomando como principio el diagnóstico psicopedagógico para mejorar el comportamiento infantil en etapa educativa inicial.

A la luz del presente problema, se vislumbra la necesidad de realizar nuevas investigaciones sobre la temática, ya que la pedagogía y la psicología presentan algunas herramientas esenciales para el maestro, que al ser aplicadas ayudaran a detectar o conocer que falencia presenta el educando y buscar soluciones.

La psicopedagogía es una rama de la psicología que se ocupa de los fenómenos de orden psicológico para llegar a una formulación más adecuada de los métodos didácticos y pedagógicos de intervención oportuna.
El diagnóstico psicopedagógico permite recopilar información de las conductas para definición de las competencias, por ende, los estados de ánimos, intenciones o problemas de una determina persona. Dentro del campo psicopedagógico se requiere del acopio de observaciones, datos e informaciones que establezca una mayor compresión especifica de una situación vital o aprendizaje de un niño, un joven o un adulto. (Gento, Trost, y Sautter, 2010). Un diagnóstico psicopedagógico oportuno por parte de la o el docente puede aportar significativamente en el desarrollo del comportamiento de los niños, potenciando el apoyo de los padres de familia o representantes.

El punto de partida siempre suele ser la conducta del individuo, al principio no se conoce bien cuál es la principal causa que genera el buen o mal comportamiento del niño; por este motivo el profesional debe utilizar algún tipo de instrumento que ayude a esclarecer la problemática.

Según Perez y García (1989) la evaluación psicopedagógica es "el acto de valorar una realidad que forma parte de un proceso, cuyos momentos previos son los de fijación de las características a valorar y la recogida de información de calidad sobre las mismas y cuyas etapas posteriores son la toma de decisiones en función de juicioemitido".

La psicopedagogía está relacionada especialmente con la psicología evolutiva (principalmente de niños y adolescentes); tiene, asimismo, una estrecha relación con los métodos y técnicas de diagnóstico psicológico; $y$, se ha de fundamentar en la psicología general y pedagogía (especialmente en la didáctica) más concretos, sus cometidos son: la evaluación de la 
madures escolar de niños y jóvenes la evaluación para el diagnóstico de las aptitudes escolares, las técnicas y métodos de enseñanza, asesoramiento y orientación de tutores, profesores, padres y alumnos, apoyo en contextos sociales y no formales." (Gento y Sánchez, 2010).

Para Mallart, (2012) es importante identificar cuatro enfoques dentro de la didáctica general en la psicopedagogía y con los modelos didácticos en general y los específicos de la Educación Especial los cuales son la perspectiva técnica, perspectiva práctica, perspectiva socio crítica y perspectiva emergente.

Seguidamente, Moreno (2005) señala que "el comportamiento es el conjunto de conductas y sus características con las que un individuo o un grupo determinado establece relaciones consigo mismos, con su entorno o contextos físicos y con su entorno humano más o menos inmediatos". (Moreno, 2005).

Lo que permite comprender y tenga mayor vigencia es la frase de Horace Walpole. "La vida es una comedia para quienes piensan y una tragedia para quienes sienten", (Rodriguez, 2012, p. 34), allí se hace alusión y dedica especialmente al tema de las emociones, especificamente cuando éstas no son controladas y originan problemas, conflictos, malos entendidos, a la parte agresiva del ser humano

Skinner consideró que su investigación sobre los programas de reforzamiento constituía el principal aporte de su obra. El gran descubrimiento del condicionamiento operante es que la conducta de un organismo cualquiera cae bajo el control de ciertos estímulos debido a sus consecuencias, y los programas de reforzamiento establecían las diferentes relaciones temporales en que podía darse dicho control. A través de ellos Skinner pudo dar cuenta de una gran cantidad de fenómenos, en particular del comportamiento humano, que caían dentro de lo que se denominaba conducta voluntaria, y se creían indeterminados. Los hallazgos de los programas de reforzamiento constituyen algunas de las pocas leyes bien establecidas e incontrovertibles de la psicología entendida como ciencia del individuo. (Plazas, 2006).

De acuerdo con la fundamentación analizada, la conducta tiene un carácter observable, cuantificable, se visualiza por medio de las acciones, emociones y los pensamientos. Los seres humanos, son seres sociales, que nacen y se relacionan unos con otros y a través de las interacciones que establece con ellos va integrándose en los distintos contextos en los cuales forma parte.

La variación de los comportamientos infantiles depende del entorno familiar, social y educativo, si es que los educandos no poseen equilibrio emocional o afectivo, lo ideal sería que el comportamiento de cada niño sea guiado con el apoyo de sus familiares, docentes, iguales, profesionales, de modo que tuviese connotaciones positivas en los diferentes lugares en los que se desenvuelva. Desde que el niño se encuentra en el vientre de su madre recibe afectividad, porque su progenitora acaricia su pancita, le comienza hablar, le canta, etc. Tanto así que desde ya el pequeño embrión va siendo estimulado poco a poco hasta que nazca.

El estudio del desarrollo afectivo se ha realizado a través del análisis de aspectos concretos del mismo, es un 
concepto muy amplio que abarca muchos aspectos de la personalidad entre los que destacan los vínculos que el niño establece (en edades que nos ocupan son el apego y la amistad), las emociones y el autoconcepto, sobre todo la vertiente afectiva es la autoestima. (Acaña y Martín, 2011).

El desarrollo socio afectivo pertenece a un crecimiento global del individuo. Permite al niño socializarse progresivamente, adaptándose al entorno en el cual forma parte, estableciendo relaciones sociales con sus demás compañeros o familiares, adquiere conductas a base de normas, valores y principios que se rigen en la sociedad, implica la construcción de su propia personalidad, identidad, autoconcepto y autoestima, vive en un entorno donde establece vínculos, expresa emociones, fundamenta la axiología y todo aquello que contribuye al bienestar y equilibrio personal.

Desde que el niño nace, es un ser social y las primeras interrelaciones que tiene es con su madre y luego con los miembros de su hogar, consecuentemente después se integra a un nuevo entorno que es la escuela, ahí el pequeño ya no está solo con los miembros de su familia, sino que ya hay otros individuos que conforman su nuevo medio, entonces comienza a relacionarse mediante el juego con los otros infantes. Las interrelaciones sociales que van ejerciendo los niños en su entorno también van de la mano con la afectividad ya que ellos trasmiten sentimientos cuando se está expresando con los demás seres de su especie.

Por ello, Acaña y Martín (2011) afirman que el "desarrollo afectivo es una dimensión madurativa del individuo referida a la formación de vínculos con otras personas, el desarrollo de emociones y sentimientos y la construcción de la personalidad propia que incluye motivaciones, intereses, autoconocimientos y autovaloración” (p.36).

La propiedad más importante del ser humano, es su capacidad de formar y mantener relaciones (familia). Estas son absolutamente necesarias para que pueda sobrevivir, aprender, trabajar, amar y procrearse, es por ello que desarrollo afectivoemocional forma parte de un conjunto de sentimientos, emociones y elementos sociales que configuran la relación del ser humano con su medio personal y social.

La familia es portadora de la antorcha de los cambios sociales y culturales. En la familia se produce la transmisión de valores, emociones, afectos, pensamientos, creencias, actitudes, usos, costumbres, tradiciones. El espejo de la familia refleja la identidad del pueblo, la identidad grupal y la identidad individual. Y esta identidad llevará al individuo a elegir diferentes caminos a lo largo de su vida. En la elección de estos caminos, el individuo se convierte en agente social y, como modelo cultural identificativo, es un ser activo en el cambio social. (Ruiz, 2004, p. 4)

Por lo es importante destacar que la afectividad sirve para conseguir el desarrollo integral del individuo, su finalidad es generar estrategias cognoscitivas para que el niño y la niña se relacionen con el mundo, por medio de la creación de vínculos afectivos, es por ello que abarca una serie de actividades que forman de manera fundamental dentro de la vida del ser humano, ya que es la primera área en desarrollarse y de ahí se desprende el resto de áreas de aprendizaje como lo son la cognitiva y la psicomotriz. 


\section{METODOLOGÍA}

Se empleó la modalidad de investigación cualitativa, la metodología utilizada fue descriptiva, deductiva e inductiva, permitió fundamentar teórica, analítica y descriptivamente el problema en estudio, además de fundamentar la propuesta, de igual manera se realizó el diagnóstico en la niña (objeto de estudio de caso) por medio de los registros anecdóticos de la niña, la escala descriptiva aplicada a la docente; y, la encuesta a la madre de familia sobre el comportamiento infantil, mismos que fueron validados por profesionales expertos en el área de investigación. La población de la cual se determinó el sujeto de estudio fue a los estudiantes de inicial 2 de la Unidad Educativa "El Carmen", provincia Manabí, Ecuador. El diagnóstico a través de la observación permitió tomar como muestra a una estudiante de 4 años de edad, de iniciales DIBZ, a, una docente y la madre de familia.

\section{DESPCRIPCIÓN Y DISCUSIÓN DEL CASO}

A través de los registros anecdóticos se pudo observar que la niña, presenta problemas en su comportamiento, de la personalidad, su autoestima es baja; en lo que respecta a su cuidado personal es desfavorable debido a que no tiene un control e higiene propia.

Estos registros permitieron también conocer en qué se estaba fallando dentro del desarrollo biopsicosocial de la niña, dentro de los tres ámbitos se encuentra presente la problemática: En el ámbito biológico, porque no siente interés por su propio cuidado e higiene personal; en el ámbito psicológico: porque se está fallando en su comportamiento y también su personalidad, y en el ámbito social: porque no se interrelaciona con otros niños en especial de su mismo género.
El área principal en la que se está fallando es la socio-afectiva, se tomar actuar con prontitud porque es la primera que los niños desarrollan, por cuanto es una de las áreas más importantes en la vida de todo individuo, en ella presentan una serie de emociones y sentimientos que les va a permitir con el pasar del tiempo ir creando su propia personalidad para desenvolverse en el entorno social.

En primer lugar, se analizó el comportamiento de la niña dentro del hogar, en donde la pequeña mostró ser un poco agresiva y también trata de imitar a su hermanito en muchos aspectos, su higiene personal es desfavorable, ya que siempre anda solo en ropa interior, sin zapatos; despeinada $\mathrm{y}$ en ocasiones sucia.

El entorno donde se encuentra la niña, es desorganizado y poco aseado, la mayor parte del tiempo pasan con una señora que los cuida porque el papá trabaja y la mamá estudia; por este motivo los niños quedan al cuidado de la señora, quien no les pone control cuando realizan los berrinches.

Ambos niños son agresivos con la señora que los cuida, hasta han llegado a pegarle, sin embargo ella no controla este tipo de problemática por sí misma, sino que más bien le dice a los padres de los niños y son ellos quienes juzgan la manera de corregirlos, otro aspecto que se ha notado es que cuando ambos padres corrigen a sus hijos lo hacen de manera muy brusca, porque los castigan con fajas o con un bollero en especial la mamá, ya que es quien se encarga de corregirlos, por este motivo los niños tienen respeto, miedo, a sus padres porque saben que si se comportan mal los castigaran, pero en cambio con la señora son muy malcriados y groseros. 
El ámbito familiar podría determinarse como falta de interrelación, comunicación, organización y disposición de tiempo de los padres hacia sus hijos, y en especial la falta de dedicación que la madre de familia le da a su hija, ya que ella es el único ejemplo femenino que tiene dentro del hogar.

\section{Escala descriptiva aplicada a la docente de la estudiante DIBZ de nivel inicial 2 de la U.E. "El Carmen"}

Cuando los niños empiezan su etapa escolar, es recomendable que se realice un diagnóstico, por medio del cual se pueden determinar algunas características relacionadas con su desarrollo integral, algunos niños llegan de sus casas con las características desarrolladas en cuanto a sus capacidades de poder entender el mundo que les rodea y relacionarse con los demás niños.

\section{Diagnóstico psicopedagógico al comportamiento infantil de parte del docente}

En las Tablas 1, 2, 3, 4 son descritos los datos arrojados del diagnóstico por parte de la docente la cual es la guía principal quien conoce, observa e interactúa con los estudiantes y pone más énfasis en aquellos pequeños que presentan algún problema académico, disciplinario o de comportamiento, por este motivo debe utilizar o diseñar herramientas para trabajar sobre estos inconvenientes, dado que la escuela se convierte en su segundo hogar, pasan gran parte de su tiempo, se interrelacionan, crean lazos de amistad con los demás niños y sobre todo existe un encuentro cercano con la docente quien es considerada como un gran ejemplo.

Ella evalúa y actúa en el desarrollo social de los niños realizando practicar sobre normas de relaciones y convivencia como: saludar, dar gracias, despedirse, etc.; amar a sus padres y su familia en general; reconocer y respetar las diferencias culturales, individuales, sentimientos, necesidades, emociones de los otros en su entorno familiar y social; adquirir hábitos de trabajo: orden, organización, iniciativa, capacidad de esfuerzo.

Dentro de la institución educativa el estudiante es el elemento fundamental, puesto que en él se centra toda la atención, los demás participantes son los directivos, docentes y la sociedad, que a su vez también cumplen roles importantes en el proceso educativo. Teniendo claro que la primera educación viene del hogar y los principales educadores son los padres, el pequeño ya ha adquirido alguna experiencia que poco a poco deberán ser pulidas o remodelas con la ayuda profesional del docente.

En cada institución educativa siempre habrá casos de niños con indisciplina o puede ser que en el hogar el pequeño se comporte de una manera y en el aula de clases de otra, dando como consecuencia dos tipos de personalidades o comportamientos infantiles diferentes 
Tabla 1. Higiene personal.

\begin{tabular}{lcc}
\hline \multicolumn{1}{c}{ Aspectos evaluativos } & Siempre & Pocas veces \\
\hline Llega a clase peinada & $\mathrm{x}$ & \\
Viene a clase con la cara limpia & $\mathrm{x}$ & $\mathrm{x}$ \\
Trae las manos limpias cuando llega de casa & & $\mathrm{x}$ \\
Tiene las uñas cortas y limpias & $\mathrm{x}$ & \\
Se lava las manos cuando regresa del servicio & $\mathrm{x}$ & \\
Se lava las manos cuando viene del receso & & \\
\hline
\end{tabular}

Nota: en esta tabla se muestra como es el desempeño de la niña en su higiene personal.

Tabla 2. Vestimenta.

\begin{tabular}{lcc}
\hline \multicolumn{1}{c}{ Aspectos evaluativos } & Siempre & Pocas veces \\
\hline Llega a clases con la ropa limpia & $\mathrm{x}$ & \\
Lleva la misma ropa durante varios días & & $\mathrm{xunc}$ \\
Cuida de la limpieza de su ropa & $\mathrm{x}$ \\
Los zapatos se encuentran lustrados y limpios & $\mathrm{x}$ \\
\hline
\end{tabular}

Nota: en esta tabla se muestra como es el desempeño de la niña en su vestimenta.

Tabla 3. Material.

\begin{tabular}{lcc}
\hline \multicolumn{1}{c}{ Aspectos evaluativos } & Siempre & Pocas veces \\
\hline Cuida el material de la clase y útiles & $\mathrm{x}$ \\
Es ordenada al momento de realizar una actividad & $\mathrm{x}$ \\
Hace el uso adecuado del material & $\mathrm{x}$ \\
Se preocupa por presentar bien bonito sus trabajo & $\mathrm{x}$ \\
\hline
\end{tabular}

Nota: en esta tabla se muestra el cumplimiento de la niña con los materiales de trabajo.

Tabla 4. Convivencia y sociafectividad.

\begin{tabular}{|c|c|c|c|}
\hline Aspectos evaluativos & Siempre & Pocas veces & Nunc \\
\hline Asiste a clases frecuentemente y de buen humor & & $\mathrm{x}$ & \\
\hline Comparte juegos con sus compañeros & & $\mathrm{x}$ & \\
\hline Pelea con frecuencia con sus compañeros & $\mathrm{x}$ & & \\
\hline Sus padres asisten con frecuencia a la institución & & $\mathrm{x}$ & \\
\hline $\begin{array}{l}\text { Utilizaría una guía de estrategias didácticas para potenciar el comportamiento infantil } \\
\text { en el desarrollo del proceso didáctico de aula. }\end{array}$ & $\mathrm{x}$ & & \\
\hline
\end{tabular}

Nota: en esta tabla se muestra como es el desempeño de la niña en convivencia y socio afectividad. 
Según los señalado en párrafos y evidencias anteriores, es necesario comprender que las emociones son inevitables, debido a que el organismo necesita liberar la parte biológica, en donde se ven manifestada la pausa, lágrimas, estar alegre, esto con la finalidad de dar respuesta ante los estímulos que, si no se tuviese ésta, no se podría responder a la interacción humana, no se sabría cuando parar, cuando sonreír y cuando amar. (Rodríguez, 2012, p. 33).

Por ello, el progreso del niño hacia el logro o desarrollo de su personalidad requiere ser influencia por los factores que se señalaron al inicio sobre el crecimiento cognoscitivo, ya que el estadio o nivel evolutivo, va permitiendo alcanzar logros y capacidades; en segundo lugar está el estilo de crianza, es decir las relaciones del niño con sus padres permiten el forjamiento o no de la personalidad, en tercer lugar está la escolaridad, donde le muestran al niño otros elementos normativos y valorativos de nivel cultural que permiten al avance y desempeño social y por ultimo está el contexto social y cultural; por ello el proceso de forjamiento de la personalidad se hace complejo para los niños, pues siempre hay una presión sobre sus actuaciones y actitudes. (Betancur, 2010, pp. 44-45).

\section{Propuesta de Estrategias Didácticas para mejorar el comportamiento infantil en los niños de educación inicial de 4 a 5 años}

La propuesta ayudará a dar una solución a la problemática señalada al inicio, ya que ofrece una serie de estrategias didácticas que contribuirán a mejorar el comportamiento infantil, fortalecer los diferentes tipos de inteligencia, elevar la autoestima y el área socio afectivo en los niños de Educación Inicial de 4 a 5 años, considerando aspectos cotidianos de la vida de ellos, su entorno familiar y educativo, involucrando a sus padres y docentes. (Estrategias propuestas ver Tabla $5,6,7,9,10,11,12,13)$.

El niño participará y se integrará en juegos, trabajos individuales y grupales; cultivo de la alegría y el buen humor. Partiendo de la concepción que desde nacimiento y los primeros 6 años el niño se forja una imagen de sí mismo. Siendo los padres el principal elemento en transmitir las herramientas necesarias para desarrollar actitudes que le permitan mantener y construir una buena autoestima (Laporte, 2002).

Tabla 5. Estrategia: Dramatización Cuento "ricitos de oro".

\begin{tabular}{|c|c|}
\hline Estrategia $N^{\circ} 1$ & $\begin{array}{l}\text { Dramatización Cuento "ricitos de oro" o se puede realizar otro de acuerdo a la temática que se esté } \\
\text { desarrollando en clase o que incluya algún valor. }\end{array}$ \\
\hline Área & Socio afectiva \\
\hline Inteligencia & Inteligencia intra e interpersonal \\
\hline Objetivo & Motivar la autovaloración a través del cuento "ricitos de oro" \\
\hline Materiales & Vestimenta, escenario, grabadora, cuento, TV, CD, DVD, Talento humano, tela, tijeras, cintas, cartulina. \\
\hline Procedimiento & $\begin{array}{l}\text { Observar el video del cuento "Ricito de oro" } \\
\text { Hacer un conversatorio del cuento. } \\
\text { Distribución de cada uno de los papeles protagonistas } \\
\text { Breve ensayo del cuento } \\
\text { Organización del escenario. } \\
\text { Realización del cuento. }\end{array}$ \\
\hline
\end{tabular}

Nota: en esta tabla consta diseño de estrategia de la propuesta didáctica. 
Tabla 6. Estrategia: Orquesta musical "caritas felices".

Estrategia No 2 La orquesta musical "caritas felices"

Área

Socio afectiva

Inteligencia Inteligencia musical, intra e interpersonal

Tiempo

20 a 30 minutos

Objetivo encuentra en el medio, para la orquesta musical.

Materiales Tarro de pintura vacío, foami, palillos, cinta, lapiceros, marcadores, lápiz de colores, sorbetes, goma, tijeras.

Procedimiento Presentar una lámina con los instrumentos musicales.

Seleccionar con los niños cuales son los instrumentos musicales que ellos quieren realizar.

Entregar el material a los niños para realizar los instrumentos.

Una vez realizados los instrumentos, seleccionar una música conjuntamente con los niños.

Se finaliza formando la orquesta y cantando la canción

Nota: en esta tabla consta diseño de estrategia de la propuesta didáctica.

Tabla 7. Estrategia: Estrategias grafo plásticas.

\begin{tabular}{ll}
\hline Estrategia No 3 & Actividades grafo plásticas "mis pequeños artistas" \\
Área & Socio afectiva \\
Inteligencia & Inteligencia espacial e interpersonal \\
Objetivo & Impulsar la creatividad de los niños a través de técnicas grafo plásticas \\
Materiales & Talento humano, granos, goma, tijeras, pintura, marcadores, lápices de colores, cintas, hojas, láminas, papelotes. \\
Procedimiento & $\begin{array}{l}\text { Los padres conjuntamente con sus hijos deben seleccionar la técnica que desean hacer. } \\
\text { Los padres deben brindar el material a cada uno de sus hijos para que ellos elaboren la técnica. }\end{array}$ \\
& $\begin{array}{l}\text { Es importante que los padres sean guías de sus hijos. } \\
\text { Una vez realizada la técnica, los padres deben hacer un cuadro para enmarcar la técnica y colocarla en la } \\
\text { pared de sus casas como una obra de arte. }\end{array}$
\end{tabular}

Nota: en eesta tabla consta diseño de estrategia de la propuesta didáctica. 
Tabla 8. Estrategia: Juego de profesiones.

\section{Estrategia No 4 Juego de profesiones}

Área Socio afectiva

Inteligencia $\quad$ Inteligencia intrapersonal e interpersonal $\quad$ Tiempo 20 a 30 minutos

Objetivo Desarrollar la motivación y la autoestima de la niña a través de lo lúdico

Materiales Láminas de las profesiones, cd, DVD, televisión, disfraces, talento humano.

Procedimiento Presentar una lámina de los tipos de profesiones.

Observar un video de las profesiones.

Preguntar a los niños que les gustaría ser cuando estén grandes.

Imitar las actividades que se realiza en cada profesión

Asignar a cada niño su profesión.

Disfrazar a cada niño de lo que ellos quieren ser.

Pedir a los niños que dramaticen la profesión que escogió

Nota: en esta tabla consta diseño de estrategia de la propuesta didáctica.

Tabla 9. Estrategia: Paseos familiares "Día de campo".

\section{Estrategia No 5 Juego de profesiones}

Área Socio afectiva

Inteligencia $\quad$ Inteligencia intrapersonal e interpersonal Tiempo Una mañana

Objetivo Motivar la afectividad de cada uno de los miembros del hogar a través de paseos familiares

Materiales Talento humano, finca - coliseo, comida, sillas, mesas, sábana, pelotas

Procedimiento Los padres de familia deben ponerse de acuerdo el lugar donde van a pasar el día de campo con sus hijos, puede ser juntos con la docente y los demás niños del salón.

Realizar una excursión para conocer el lugar donde se encuentran

Realizar actividades lúdicas donde participen todos los miembros de las familias como jugar futbol, u otros juegos.

Posteriormente todos deben ayudar a la hora de servir los alimentos.

Es importante hacer una sección de fotos como recuerdo.

Nota: en esta tabla consta diseño de estrategia de la propuesta didáctica. 
Tabla 10. Estrategia: Paseos familiares "Día de ejercicios en la playa".

\begin{tabular}{ll}
\hline Estrategia No $\mathbf{6}$ & Paseos familiares "Día de ejercicios en la playa" \\
Área & Socio afectiva \\
Inteligencia & Inteligencia corporal kinestésica, intra e interpersonal \\
Objetivo & Crear actividades caporales a través del juego para fortalecer los lazos afectivos de la familia. \\
Materiales & Talento humano, playa, comida, ropa adecuada, pelotas cuerdas, juguetes. \\
Procedimiento & Los padres de familia deben ponerse de acuerdo el lugar donde van a pasar el día de playa con sus hijos. \\
& Iniciar jugando con arena y construyendo castillos o diseñando otro tipo de objeto. \\
& Jugar con la pelota todos los miembros de la familia. \\
& Se debe realizar actividades como estilos circuitos. \\
& Luego jugar con la cuerda. \\
& Posteriormente bañarse en la playa en familia. \\
& Es importante hacer una sección de fotos como recuerdo.
\end{tabular}

Nota: en esta tabla consta diseño de estrategia de la propuesta didáctica.

Tabla 11. Estrategia: Paseos familiares "Juguemos a la casita de muñecas".

Estrategia No 7 Juguemos a la casita de muñecas

Área Socio afectiva

Inteligencia $\quad$ Inteligencia intrapersonal e interpersonal $\quad$ Tiempo 30 minutos

Objetivo Motivar la autoestima de la niña a través de actividades lúdicas

Materiales Talento humano, casita, muñecas, ropa, juguetes.

Procedimiento La mamá debe motivar a la hija a jugar, uno de los juegos que más gusta a las niñas son las muñecas.

La mamá participa de la actividad lúdica con las muñecas.

Debe de mostrarle a su hija que ella puede confiar.

También es importante que ella le enseñe como jugar.

Luego proceda a enseñar normas de cuidado e higiene, orden, disciplina, para que la niña conozca la importancia de aquello.

Luego invitar a otras niñas a que jueguen.

Puede repetir la actividad tantas veces que desee.

Nota: en esta tabla consta diseño de estrategia de la propuesta didáctica. 
Tabla 12. Estrategia: "Yo y mi conciencia".

\section{Estrategia No 8 Yo y mi conciencia}

Área Socio afectiva

Inteligencia Inteligencia intrapersonal

Tiempo 30 minutos

Objetivo $\quad$ Fortalecer el desarrollo de la autoconciencia

Materiales Talento humano, espejo

Procedimiento Se invita a los niños a realizar diferentes tipos de muecas frente al espejo, especialmente escenas de ira, enojo, llanto, tristeza u otros estados emocionales.

Luego preguntarle cuáles de los rostros le fueron más agradables y cuáles eran más acertados para acercarse a otros niños,

Realizar ensayos sobre el mejor rostro para acercarse a otros niños.

Nota: en esta tabla consta diseño de estrategia de la propuesta didáctica.

Tabla 13. Estrategia: "Mi compañera y yo".

Estrategia No 9 Mi compañero y yo

Área Socio afectiva

Inteligencia $\quad$ Inteligencia intrapersonal e interpersonal $\quad$ Tiempo 30 minutos

Objetivo Desarrollar la aceptación de los compañeros de sus virtudes y defectos.

Materiales Talento humano, espejo

Procedimiento Se dividirá a los niños en dos grupos

Se colocará los dos grupos uno frente al otro.

Los niños se saludarán y los del frente les dirán cosas positivas a los niños de enfrente como: eres muy alegre, eres un buen amigo, eres genial, etcétera y el compañero de enfrente dirá lo mismo.

Una vez concluida esta etapa los niños procederán a decirse entre ellos elementos positivos respecto a su cuerpo: tienes bonitos ojos, tu cabello es hermoso, tienes linda sonrisa.

Todo esto con la intención de elevar la autoestima de los niños y también de fomentar en ellos el control emocional al escuchar características físicas y psicológicas de los compañeros.

Nota: en esta tabla consta diseño de estrategia de la propuesta didáctica. 


\section{CONCLUSIONES}

Luegodelanálisisderesultadosdelainvestigación de campo se llegó a las siguientes conclusiones: Se determina la notable importancia del diagnóstico psicopedagógico en el comportamiento infantil en el subnivel inicial 2, porque es un proceso imprescindible para el conocimiento de la realidad y el comportamiento infantil, aspectos que quizás pasan de desapercibido y que son de gran importancia en el desarrollo integral, tal es el caso de la niña DIBZ, en este caso y otros similares es necesario desarrollar una apropiada comunicación entre padres o representantes y docentes con el fin de lograr su apoyo didáctico y acompañamiento para superar estas dificultades, que si no se tratan a tiempo se pueden prolongar a medida que transcurre el tiempo y por último motivar a los padres que les dediquen tiempo de calidad, que les presten más atención, que potencien el área socio afectiva, tomen otras medidas para corregir a sus hijos ya que si utilizan el mismo método reforzaran el comportamiento agresivo. Se sustentó la bibliografía de las variables que intervienen en la problemática de estudio, y se evidenció la estrecha relación que existe en las dos, como es el diagnóstico psicopedagógico y el comportamiento infantil. Las herramientas que utiliza el diagnóstico psicopedagógico son fundamentales para conocer el tipo de problemática que presentan los niños, para este trabajo se utilizó el registro anecdótico, la ficha descriptiva yelcuestionario, las quehan aportadocon una serie aspectos que proporcionaron información al informe final, se hace necesario utilizarlas para conocer la realidad de los estudiantes, abordarlos oportunamente y aplicar estrategias didácticas que ayuden a erradicar o disminuir la problemática.
La detección de la realidad y el comportamiento de DIBZ, afloró deficiencias en normas de conducta, de convivencia, disciplinarias; falta de hábitos de limpieza, poca atención por parte de los padres. El entorno familiar es la base fundamental para los niños, ya que una buena interrelación, comunicación y los valores de los padres, formarán niños con una buena autoestima, personalidad positiva y sobre todo niños seguros para desempeñarse en el ámbito social. Existe la predisposición de los docentes de ajustar al proceso educativo estrategias didácticas para mejorar el comportamiento de sus estudiantes, que con la creatividad de ellos pueden ser adaptadas, corregidas o reforzadas, por lo cual se elaboró una Propuesta de Estrategias Didácticas para mejorar el comportamiento infantil en los niños de educación inicial de 4 a 5 años.

\section{REFERENCIAS}

Acaña, L., y Martín, N. (2011). Desarrollo Socio Afectivo. España: Ma José López Raso

Betancur, T. (2010). La interacción entre niños de 3 a 5 años durante los procesos de socialización. Caldas: http://repository.lasallista.edu.co/ dspace/bitstream/10567/649/1/Interaccion_ ninos_3_a_5_anos_procesos_de_socializacion. pdf

Gento, S., y Sánchez, E. (2010). Bases Neurológicas y Psicopedagógicas del Tratamiento Educativo de la Diversidad. Madrid: Uned estudios

Gento, S., Trost, R., \& Sautter, H. (2010). Evaluación $y$ estimación del rendimiento en el tratamiento eductivo de la diversidad. Madrid: Uned Estudios

Laporte, D. (2002). Escuela para padres de 0 a 6 años: Cómo Desarrollar la Asutoestima en los niños de 0 a 6 años. Danielle Laporte: Quarzo https:// www.goodreads.com/book/show/2308805. Escuela_Para_Padres_0_A_6_Anos

Mallart, J. (2012). Respuestas de investigadores del área de Didáctica y Organización Escolar 
Mora, J. (1998). Acción tutorial y orientación educativa. Madrid: Narcea S.A

Moreno, F. (2005). Los problemas del comportamiento en el contexto escolar. Departamento de Pedagogía aplicada. Barcelona - España: universidad Autónoma de Barcelona

Morrison, G. (2005). Educación Infantil. Madrid: Pearson Educación, S. A

Perez, R., y García, J. (1989). Diagnóstico, evaluación $y$ toma de decisiones. Madrid - España: Rialp

Plazas, E. (2006). La búsqueda de orden en la conducta voluntaria. Universias Psycologicas, 7 de 14 http://www.scielo.org.co/pdf/rups/v5n2/ v5n2a13.pdf
Rodriguez, C. (2012). Psicología Social. Tlalnepantla - EStado de México: Red TErcer Milenio http://fundacionortizavila.com/descargar/340/ bc67b32d67716a4ee6dcfbc77e0ef173

Ruiz, C. (2004). El papel de la familia en la transmisión sociocultural y de la salud mental. Madrid - España: Nómadas núm. 9 https:// www.redalyc.org/articulo.oa?id=18100924 